\title{
Artificial intelligence, public control, and supply of a vital commodity like COVID-19 vaccine
}

\author{
Vladimir Tsyganov ${ }^{1}$
}

Received: 24 April 2021 / Accepted: 3 September 2021

(c) The Author(s), under exclusive licence to Springer-Verlag London Ltd., part of Springer Nature 2021

\begin{abstract}
The article examines the problem of ensuring the political stability of a democratic social system with a shortage of a vital commodity (like vaccine against COVID-19). In such a system, members of society citizens assess the authorities. Thus, actions by the authorities to increase the supply of this commodity can contribute to citizens' approval and hence political stability. However, this supply is influenced by random factors, the actions of competitors, etc. Therefore, citizens do not have sufficient information about all the possibilities of supplying, and it is difficult for them to make the right decisions. Such citizen unawareness can be exploited by unscrupulous politicians to achieve personal targets. Therefore, it is necessary to organize public control to motivate politicians to use all available opportunities in supplying. The goal of the paper is to build such a digital mechanism of public control of the politicians by citizens, which would best assess and stimulate the activities of the authorities to improve the supply of a vital commodity. In the age of artificial intelligence, such digital public control in the face of uncertainty can be based on digital machine learning. In addition, it is necessary to take into account and model the activities of politicians associated with the presence of their own targets that do not coincide with public ones. Such politicians can use the learning of citizens for their own targets. The objective of the article is to build an optimal digital mechanism of public control in a two-level model of a democratic social system - a digital society. At its top level, there is the Citizen, who gives an assessment for the Politico located at the lower level. In turn, the Politico can influence the supplying of a vital commodity. Political stability is guaranteed if the Citizen regularly approves of the Politico's actions to increase this supply. However, the Politico may not use the opportunities available to him to offer a commodity to achieve a personal target. To avoid this, the Politico's control mechanism is proposed. It includes the procedure for digital learning of the Citizen, as well as a procedure for assessing the Politico activity. Sufficient conditions have been found for the synthesis of such the Politico's control mechanism, at which stochastic possibilities of increasing the supply of a vital commodity are used. The example of such the Politico's control mechanism is considered on the case of supply of the COVID-19 vaccine in England.
\end{abstract}

Keywords Social system $\cdot$ Political stability $\cdot$ Digital $\cdot$ Society $\cdot$ Control $\cdot$ AI $\cdot$ Machine learning $\cdot$ COVID-19 $\cdot$ Vaccine

\section{Introduction}

\subsection{Democratic system, political stability, and public control}

In the face of a shortage of a vital commodity such as the vaccine against COVID-19 (briefly vaccine), the authorities must take the job of monitoring and supporting its supply

Vladimir Tsyganov

bbc@ipu.ru

1 Institute of Control Sciences, Russian Academy of Sciences, 65 Profsoyuznaya Street, Moscow 117997, Russia in the right quantities. Therefore, authorities need to control the supply of this commodity (briefly supply), in the face of change and associated uncertainty. This concerns the development and production at factories that are located in the territory they control. The authorities also have other opportunities to increase the supply, for example, through procurement from the external market or through production under license in the economic system they control.

Unfortunately, however, market fundamentalism leads to a crisis of values of politicians and their attempts to pursue personal rather than public goals (Soros 1998). Therefore, it is necessary to organize public control to motivate such unscrupulous authorities to use all available opportunities 
to overcome the social crisis. Lack of proper social control leads to the emergence of toxic leaders and corrupt politicians (Lipman-Blumen 2004), oligarchy (Schultz and Tsyganov 2009), leaders without ethics (Boddy et al. 2010), hedonistic politicians (Tsyganov 2014), and wirepullers creating phobias (Tsyganov 2020).

In a democracy, society controls politicians through elections, politicians' ratings, protests, etc. Therefore, political stability is fostered by public acceptance of politician actions to secure supplies of a vital commodity such as the vaccine. Taking into account the above, we will consider the hierarchical structure of a public control in the model of sociopolitical system "Society-Power", as shown in Fig. 1a).

In the face of uncertainty, public control over the supply of a vital commodity (like vaccine) can be based on artificial intelligence (AI). AI is already contributing to the fight against COVID-19. Let us consider the main directions of AI applications in this area.

\subsection{Artificial intelligence vs COVID-19}

During the COVID-19 pandemic (briefly Pandemic), there have been various attempts at AI deployment, mainly in the bio-medical field. AI applications in biological and medical informatics aim to improve the diagnosis and treatment of COVID-19 disease. Nguyen (2020) provided an overview of AI techniques used in the Pandemic, from medical imaging, data analysis, text mining, and natural language processing, the Internet of Things to computational biology and medicine. Ahuja et al. (2020) considered development, testing, and approval process of medical remedies and vaccines.

Tayarani (2021) reviewed the use of AI, including diagnosing disease through various tests and symptoms, observing patients, determining patient severity, processing imaging tests, epidemiology, and pharmaceutical research. Alazab et al. (2020) reviewed COVID-1019 prediction and detection methods using deep machine learning (ML).

Jamshidi et al. (2020) describe an integrated bioinformatics approach that combines information from structured and unstructured data sources to form convenient platforms for clinicians and researchers. Such AI-based platforms are accelerating the diagnosis and treatment of COVID-19 disease. Islam et al. (2020) developed smart healthcare monitoring system in Internet of Things environment.

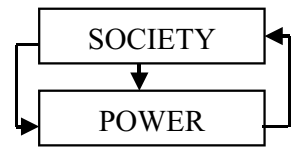

a

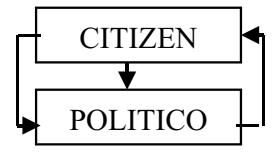

b
Fig. 1 Hierarchical structures of socio-political systems: a SocietyPower. b Citizen-Politico
Abdulkareem and Petersen (2021) described AI systems used to diagnose COVID-19 through medical imaging, such as computed tomography of the chest and X-rays. These AI systems differ in algorithms for image segmentation, classification, and diagnosis of diseases. Mohammad-Rahimi et al. (2021) review application of ML in diagnosis of COVID-19 through X-ray and computed tomography images.

AI applications in socio-medical informatics and engineering aim to improve epidemiological forecasting and social control. Shinde et al. (2020) made a survey of forecasting models for COVID-19 disease using ML. Abdulkareem and Petersen (2021) described AI methods used to predict mortality rates, length of hospital stay, and outcomes for COVID-19 patients. Ahmed et al. (2021) provided ML platform for tracking social distance. Ahuja et al. (2020) considered multidisciplinary approach to AI and COVID-19, whereby AI techniques can help to disseminate vital information and reduce the spread of false information about COVID-19.

However, the attempts to apply AI in the social domain such as tracing and tracking of infections to halt uncontrolled spread have met with open scepticism. Note that the cited above AI studies on COVID-19 is based on statistical approaches. As with virtually all known AI research in this area, these approaches depend solely on correlation factors. The causality factors were not considered, because there were no formal models based on causality. However, correlation does not imply causality, while causality always implies correlation (Groumpos, 2021).

For his part, Naudé (2020) concluded that AI has yet to make an impact on the Pandemic, either due to insufficient data or too much data. One of the reasons for this is the failure to take into account the causal relationships between different data. In this regard, the approach of Fuzzy Cognitive Maps (FCM) was proposed for the study of COVID19 , taking into account the factors of causality (Groumpos, 2021). Until now, this FCM approach has probably been the only one that investigated the causal relationship between the variables of medical problem of COVID-19. In connection with the above, a hybrid approach to AI research in the field of COVID-19, based on statistics and correlation, as well as mathematical modeling and causality, is of particular interest.

Naudé (2020) concluded that overcoming constraints between a lack of data, and by too much data will require a careful balance between data privacy and public health, and rigorous human-AI interaction. In turn, this is associated with more general questions of using $\mathrm{AI}$ in society.

\subsection{Questions of using Al in society}

The World Economic Forum White Paper (2019) concludes that "The increasing use of AI ... will have revolutionary 
effects on human society ... thereby preserve a human-centric society." AI makes it possible to implement the prediction paradigm "as a technological dream of public safety, national security" (Gill 2020a).

However, there are difficulties on the way to the effective use of AI in society. First of all, there is a need of algorithmic accountability and transparency in the use of AI for making public decisions (De Fine Licht et al. 2020). It is necessary "to initiate a conversation on public accountability frameworks, including issues of governance, and the cultivation of a culture of algorithmic accountability arising from concerns of opaqueness, transparency and responsibility" (Gill 2020b).

In seeking an insight into issues of accountability, de Laat (2018) explores issues of opaqueness, transparency, and responsibility of algorithmic systems and asks "How to install a culture of accountability?". He wonders whether we should follow the "dominant trajectory of developing algorithms that become ever more accurate but ever less intelligible?" or we should follow the trajectory of seeking explanations for algorithmic decisions? He concludes that by opting for the trajectory of explanation, we may expect "full accountability" but with the limited amount of transparency in algorithmic decision-making, "given the state of the art in machine learning and the current political climate." In this regard, Gill (2020b) asks the question: "What should be our response to AI Agency trapping us in the data-driven web of the AI-powered machine?".

Spiegelhalter (2020) says that perhaps a more basic issue is whether we should believe what we hear about AI and what the algorithm tells us. For those interested in the design of trustworthiness of AI algorithms, he proposes seven criteria for the evaluation of claims of trustworthiness: (1) is it any good when tried in new parts of the real world? (2) Would something simpler, and more transparent and robust, be just as good? (3) Could I explain how it works (in general) to anyone who is interested? (4) Could I explain to an individual how it reached its conclusion in their particular case? (5) Does it know when it is on shaky ground, and can it acknowledge uncertainty? (6) Do people use it appropriately, with the right level of skepticism? (7) Does it actually help in practice? In seeking trustworthiness of AI system, he asks to be mindful in that "It is important not to be mesmerised by the mystique surrounding AI."

\subsection{Human factor}

In addition to the above, the use of AI is hampered by the gap between ML and control theory (Recht 2020). It is also necessary to take into account the activity of the elements of the socio-political systems associated with the presence of their own goals. For their part, as mentioned above, the authorities may not fully use all the opportunities of supply - purchases of a vital commodity in foreign markets, acquisition of production licenses, etc. Indeed, in the face of changes, a politician knows its capabilities better than a citizen. Using this insight, the politician can manipulate. Tsyganov (2020) called such a politician the Politico. His unwanted activity can lead to the failure to use existing opportunities in which a citizen is interested. In this regard, there is a need to control and evaluate the actions of the authorities on the part of society.

Thus, to ensure political stability in a social crisis caused by a shortage of a vital commodity, it is necessary to take into account the human factor. For this, theory of organizational control is traditionally used (Burkov et al. 2013). One of the directions in this theory is associated with learning and adaptation. On their basis, adaptive mechanisms for improving international stability developed (Bagamaev et al. 2005). Arifovic and Ledyard (2011) derived a behavioral model for control mechanism design including individual evolutionary learning.

Particular attention is directed toward adaptations of the widely used self-learning algorithms to increase the efficiency of control with the aid of AI methodology. Drawing from the experience gained in the implementation of adaptive control in large-scale socio-economic systems, multilevel self-learning and self-organization have been proposed (Tsyganov, 2019). There, an integration of different learning and control systems is based on adaptive archetypes used to design adaptive information models. This approach is similar to systems engineering (Kossiakoff et al. 2011).

\section{The main assumptions in investigated model}

Critical questions like the issue of privacy, bias, transparency, and accountability of AI algorithms still remain unanswered. Therefore, deployment of AI in the public domain warrants extreme caution-especially since a "culture of algorithmic accountability" has yet to be properly established. For these reasons, before moving on to a highly formalized discussion of the technical and computational problems of digital learning and control in the supply of a vital commodity, consider the main assumptions in the investigated model.

\subsection{Model of a digital society}

In the long term, AI will become the prerogative of not only the authorities, but also members of society. Related to AI is the concept of a digital society adopted by the European Commission to access citizens to better social care, health monitoring, etc. (EU Homepage 2020). For example, 
Tsyganov (2020) developed models of voters estimated politicians in a digital society under uncertainty.

For simplicity, we will assume that citizens in a digital society learn and make decisions independently using the same optimal procedure of ML. Then, without limiting the generality, it is enough to consider a socio-political system consisting of one citizen and one politician (Fig. 1b). At the top level of its organizational structure is a member of a digital society (Citizen). At the lower level, there is a representative of the authorities responsible for supplying the Citizen with a vital commodity (Politico).

In line with the concept of a digital society (EU Homepage 2020), we will assume that both the Citizen and the Politico are using appropriate digital procedures based on quantitative data. This requires digital models of these persons. Let us consider the existing prerequisites for the construction of such models.

The social and rational models of personality are widely known, the origin of which goes back to the Nobel laureate Simon (1966). Also known models of human behavior, derived at the intersection of mathematical psychology and economics. They are based on game theory and are widely recognized in the scientific community. These include theory of prospects (the Nobel Prizes laureate Tversky and Kahneman 1992), dedicated to the expectations of economic agents.

A new class of digital models of person is based on its psychology and physiology. With their help, the dependence of human behavior on hormonal characteristics is investigated (Fehr and Rangel 2011). Based on this, humanitarian high technologies in political system of society were proposed (Schultz and Tsyganov 2012). These technologies were generalized to the cases of persons, which are controlled both by desires and by fears (Tsyganov 2020).

In addition to the above, models of members of a digital society must take into account their ability to learn, adapt, and use digital decision-making in the face of uncertainty.

\subsection{Model of the Citizen}

When forming the Citizen model, we proceed from the following assumptions. First, the Citizen is interested in increasing a certain digital indicator characterizing the effectiveness of a politician's activities in supplying society with a vital commodity. For example, in the Pandemic, the number of vaccines supplied by the authorities to vaccinate members of society can serve as such a digital indicator. The Citizen who is afraid of getting sick is interested in increasing this indicator, since this reduces the risk of his infection from others.

Second, to motivate a politician to improve efficiency, the Citizen evaluates politician's activities using regularly calculated ratings. Namely, in each period, the Citizen gives the Politico the first rating if the efficiency indicator of the Politico in supplying society with a vital commodity is not lower than a certain norm (or zeroth rating if not). For example, in the Pandemic, such supply norm may be a reasonable (from the Citizen's point of view) amount of vaccines that the authorities must provide to vaccinate members of the society.

Third, when forming the supply norm, the reasonable Citizen takes into account that supply of a vital commodity is affected by random environmental factors. Therefore, the Citizen does not know the maximum possibilities of commodity supply under the circumstances. Thus, to form the specified norm for assessing the activities of the Politico in the face of such uncertainty, the Citizen must use an appropriate digital learning procedure belongs to the class of ML procedures.

\subsection{Model of the Politico}

The authorities are more informed about the supply opportunities than citizens and other stakeholders. Auster (2013) calls such cases asymmetric awareness of the parties. Then, the problem arises of the Politico disinterest in using all the possibilities in the interests of the Citizen. This problem is typical of political systems (Soros 1998). As a result, "estimations of policy effectiveness fail to account for dynamics that are fundamental in human life and central to many public policy challenges" (Diallo et al. 2021).

With this in mind, it is needed to develop digital public policy control using AI and ML. Tsyganov (2020) considered a model of supervised learning and voter decisionmaking on voting for or against a politician in a digital society. With insufficient voter awareness, an unscrupulous politician may not use the available opportunities to achieve his goals. To avoid this, the voter learns using the recommendations of a mentor (who also does not know about all the possibilities of politician). Then, the voter uses obtained knowledge to evaluate and vote under conditions of uncertainty caused by both random influences of the external environment and undesirable activities of a politician. The evaluation and voting procedure constitutes the mechanism for making a decision by the voter. With this mechanism, a set of politician's choices is determined at which the maximum of his objective function is achieved. Sufficient conditions have been found for the synthesis of an optimal mechanism in which a politician fully uses his capabilities in the interests of the voter.

We will assume that the Politico is interested in increasing his current and future digital ratings. To achieve this, it can use the available possibilities of commodity supply. 


\section{Digital citizen learning and control of the Politico}

\subsection{The citizen self-learning}

Taking assumptions described in Sect. 2, consider a mathematical model of digital Citizen learning for evaluating the Politico actions to increase the supply. Let us denote by $t$ the period of time, $t=1,2 \ldots$. Also denote the supply, expressed in terms of the number of commodity units in the period $t$, as $q_{t}, 0 \leq q_{t} \leq Q_{t}$. There, $Q_{t}$ is the maximum possible supply, depending on random factors, $0 \leq Q_{t} \leq F$. Therefore, we will assume that $Q_{t}$ is a stationary stochastic variable.

In general, the Citizen does not know $Q_{t}$. With such uncertainty, the Citizen must classify the Politico to stimulate an increase in the total supply $q_{t}$ to value $Q_{t}$. The simplest classification is based on dichotomy, i.e., assigning the Politico one of two ratings - 0 or 1 (see Sect. 2.2). Namely, the Citizen gives the Politico first rating if the Politico work for supplying is satisfactory (or zeroth rating if not). For example, if the Politico gets first rating then the Citizen votes for him. Otherwise (zeroth rating), the Citizen does not vote for the Politico.

Consider the formal setting and solution of the dichotomy task, i.e., assigning the Politico one of two ratings - 0 or 1. First, suppose the Citizen knows the maximum possible supply $Q_{t}$. To determine the rating of the Politico in period $t$, it is necessary to assign supply $Q_{t}$ to one of the two sets $\Pi_{1}$ and $\Pi_{2}$ which make up the set $\Pi, \Pi=[0, F]$, $\Pi_{1} \cup \Pi_{2}=\Pi$ If $Q_{t} \in \Pi_{1}$, then the Citizen gives the Politico zeroth rating, otherwise-1st rating. Taking into account $0 \leq Q_{t} \leq F$, this means that to determine the Politico's rating, the Citizen must divide the segment $[0, F]$ into interval $[0, p)$ and segment $[p, F]$. Here, $p$ is the parameter used when deciding on the Politico's rating: if $Q_{t}<p$ then the Citizen gives the Politico zeroth rating. Otherwise, if $Q_{t} \geq p$, then the Politico obtains first rating. Essentially, $p$ is the minimum quantity of a vital commodity that the Politico must supply in order to receive first rating.

Assigned incorrect rating leads to losses of the Citizen. The task is to determine such a division of the set $\Pi$ that minimizes the average losses from the incorrect dichotomous classification of the Politico. Then, for each unknown set $\Pi_{1}$ and $\Pi_{2}$, the Citizen can introduce loss functions, as dependences of losses on incorrect classification:

- $L_{1}\left(c, Q_{t}\right)$-losses in case if the Citizen believes that $Q_{t} \in \Pi_{2}$, and gives the Politico first rating (i.e., the Politico work for supplying is considered as satisfactory), while in fact it is not: $Q_{t} \in \Pi_{1}$ (that is, this work is unsatisfactory);
- $L_{2}\left(c, Q_{t}\right)$-losses in case if the Citizen believes that $Q_{t} \in \Pi_{1}$, and gives the Politico zeroth rating (i.e., the Politico work is considered as non-satisfactory), while $Q_{t} \in \Pi_{2}$ (i.e., this work is satisfactory).

We will call $L_{1}\left(c, Q_{t}\right)$ and $L_{2}\left(c, Q_{t}\right)$, respectively, losses of the first and second kind. For simplicity, we will assume that those loss functions are linear.

$L_{1}\left(c, Q_{t}\right)=Q_{t}-c m, \quad L_{2}\left(c, Q_{t}\right)=f\left(c-Q_{t}\right)$,

where $c$ is an unknown parameter calculated so as to minimize the average losses of classification, and $m$ and $f$ are coefficients characterizing the ratio of losses of the first and second kind, respectively, $0<m<1, f>0$. For obtaining the best estimate $c_{t}$ of a parameter $c$ in the dichotomy task, the optimal algorithm of digital self-learning was developed, which is described in the following Statement.

Statement 1 (Tsyganov 2019). To minimize the average losses of dichotomous classification with linear loss functions (1), it is enough for the estimates $c_{\tau}, \tau=1,2 \ldots$, to be calculated using the recurrent equation

$$
\begin{aligned}
& c_{t+1}=Z\left(c_{t}, Q_{t}\right)=\left\{\begin{array}{ll}
c_{t}+\gamma_{t} m & \text { if } Q_{t}<P_{t} \\
c_{t}-\gamma_{t} f & \text { if } Q_{t} \geq P_{t}
\end{array}, \quad t=1,2 \ldots\right. \\
& P_{t}=c_{t}(f+m) /(f+1), \quad c_{1}=c^{1} \text {, }
\end{aligned}
$$

where $\gamma_{t}$ is the adaptation coefficient, $0<\gamma_{t+1}<\gamma_{t}$, $\sum_{\tau=1}^{\infty} \gamma_{\tau}<\infty$. In an absolutely optimal algorithm that provides the maximum rate of convergence of estimates (2), $\gamma_{t}=1 / t, \quad t=1,2, \ldots$

Now, suppose the Citizen only knows the supply $q_{t}$ determined by the Politico. Then, for current estimating of $c_{t}$, the Citizen can use the algorithm of self-learning (2), in which we must substitute $q_{t}$ instead of $Q_{t}$. Then, the estimate $z_{t}$ of an unknown parameter $c$ can be calculated by the algorithm of self-learning

$$
\begin{aligned}
& z_{t+1}=Z\left(z_{t}, q_{t}\right)=\left\{\begin{array}{cc}
z_{t}+\gamma_{t} m & \text { if } q_{t}<p_{t} \\
z_{t}-\gamma_{t} f & \text { if } q_{t} \geq p_{t}
\end{array}, \quad t=1,2 \ldots\right. \\
& p_{t}=z_{t}(f+m) /(f+1), \\
& z_{1}=c^{1} .
\end{aligned}
$$

Using (3), the Citizen determines the Politico rating

$r_{t}=R\left(q_{t}, p_{t}\right)=\left\{\begin{array}{lll}1 & \text { if } & q_{t} \geq p_{t} \\ 0 & \text { if } & q_{t}<p_{t}\end{array}\right.$.

If the real supply $q_{t}$ is not lower than $p_{t}\left(q_{t} \geq p_{t}\right)$ then the Politico gets a rating $1\left(r_{t}=1\right)$; otherwise, rating zero $\left(r_{t}=0\right)$. In the other words, in case $q_{t} \geq p$, the Citizen approves the Politico activity in increasing the supply in period $t$. In case of zero rating, the Citizen can accuse the 
Politico, voted and protested against him, etc. Therefore, the support of the Politico from the Citizen rises with this rating. Thus, $p_{t}$ is the minimum quantity of a vital commodity that the Politico must supply to receive support from the Citizen. For brevity, we will call $p_{t}$ the supply norm, $Z\left(z_{t}, q_{t}\right)$-rationing procedure, and $R\left(z_{t}, q_{t}\right)$ - the rating procedure.

\subsection{The Politico choice and control mechanism}

Assume that maximal supply $Q_{t}$ becomes known to the Politico before selecting $q_{t}$. Then, the Politico chooses such $q_{t}^{*}$ which maximizing its target function depending both on the current rating and $\mu$ future ratings

$W_{t}=W\left[r_{t}, r_{t+1}, \ldots, r_{t+\mu}\right], \quad W_{t} \uparrow r_{i}, \quad i=\overline{1, \mu}$,

where $W[\cdot]$ is a non-decreasing function. Below, we assume the Politico supportive of the Citizen: if $Q_{t} \in \underset{0 \leq q_{t} \leq Q_{t}}{\operatorname{Arg} \max } W_{t}$, then $q_{t}^{*}=Q_{t}$. In essence, this means that the Politico will only understate the supply $q_{t}$ if it is beneficial to it.

The set of self-learning rationing procedure $Z\left(z_{t}, q_{t}\right)$ and rating procedure $R\left(q_{t}, p_{t}\right)$ will be called the Politico's control mechanism and denoted $P=(Z, R)$. The place of this mechanism in the considered model of digital society is illustrated in Fig. 2.

\section{Optimal Politico's control mechanism}

Consider the problem of synthesizing the optimal Politico's control mechanism $P=(Z, R)$ in which the supply of a vital commodity is maximized: $q_{t}^{*}=Q_{t}, t=1,2 \ldots$

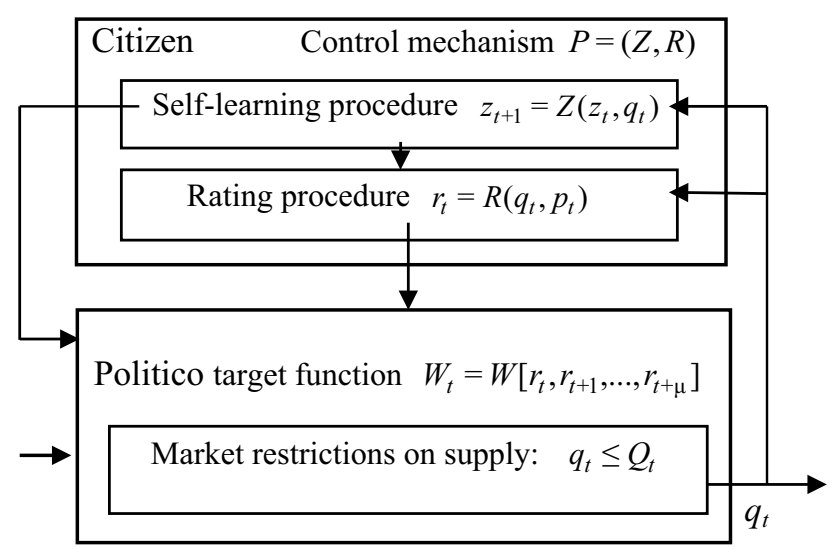

Fig. 2 The Politico's control mechanism in hierarchical social structure "Citizen - Politico"
Statement 2 The Politico's control mechanism $P=(Z, R)$ is optimal, ensuring the maximum possible vital commodity supply: $q_{t}^{*}=Q_{t}, t=1,2 \ldots$, as well as minimizing the average losses of the Politico's dichotomous classification.

Proof According to (4), the rating $r_{t}=R\left(q_{t}, p_{t}\right)$ is nondecreasing function of $q_{t}$. From (3), $z_{\tau}$ does not increase with growth $q_{t}$ when $\tau=\overline{t+1, t+\mu}$. Consequently by (4), the future rating $r_{\tau}=R\left(q_{\tau}, p_{\tau}\right)$ do not decrease with growth $q_{t}$ when $\tau=t+1, t+\mu$. Therefore, target function (5) do not decrease with growth $q_{t}$. Due to assumption that the Politico supportive of the Citizen, we have $q_{t}^{*}=Q_{t}, t=1,2 \ldots$

Furthermore, rationing procedure (3) is used to obtain the estimate $z_{t}$ in mechanism $P=(Z, R)$. However, at $q_{t}^{*}=Q_{t}$, $t=1,2 \ldots$, procedure (3) coincides with procedure (2). Therefore, estimate $z_{t}$ is equal to estimate $c_{t}: z_{t}=c_{t} t=1,2 \ldots$ However, according to Statement 1 , when using estimates of $c_{t}, t=1,2 \ldots$, the average losses of dichotomous classification with linear loss functions (1) are minimal. Therefore, estimates $z_{t} t=1,2 \ldots$, obtained with mechanism $P=(Z, R)$ minimize the average losses of the Politico's classification, QED.

Essentially, above we considered model of a digital society and the behavior of its main elements: citizens and politicians in a situation of crisis and uncertainty. Statement 2 shows that in this situation, the optimal digital strategy for every citizen is to use procedures of rationing (3) and rating (4) to classify the Politico activities. This strategy minimizes the average citizen losses when categorizing Politico. Then, Politico's optimal strategy is to maximize supply. This helps to avoid citizen discontent and possible political instability. Thus, appropriate application of AI with digital learning (i.e., ML) can reduce uncertainty and maintain political stability.

\section{Public control mechanism for COVID-19 vaccine supply in England}

Let us see how the AI-driven approach developed above can be applied to public control of vaccinations in early 2021 in England.

\subsection{The Politico's control mechanism}

The authorities have different opportunities to increase the supply of vaccine, for example, by purchasing it on the foreign market. In this regard, the problem of designing a mechanism arises, which would interest the politician in using all its available opportunities for the benefit of society. For this, the Politico's control mechanism $P=(Z, R)$ satisfying conditions of Statements can be used. 
Let us illustrate this mechanism functioning with the example of the primary vaccination against COVID-19, carried out in England from January 10 to March 31, 2021 (GOV.UK, 2021). During this period, the population's need for primary vaccination generally exceeded the corresponding supply of vaccine doses. Thus, all correspondent vaccine supplies were used for this vaccination. For this reason, the number of doses of vaccine delivered can be estimated by the number of people primary vaccinated during this period.

Formally, a member of society (Citizen) could daily observe the vaccination data $q_{t}$ which is equal to the number of people vaccinated in day $t$, where $t$ is the day and month of 2021 (black line on Fig. 3a). Then, the Citizen could use the Politico's control mechanism $P=(Z, R)$ to control vaccine supply with the procedures of rationing (2) and rating (3). The results of this mechanism functioning are illustrated in Fig. 3. The Citizen is calculating norm $p_{t}$ using (2) (blue line on Fig. 3a). The daily rating of the Politico $r_{t}$ (pink line on Fig. 3b) is determined by comparing the daily vaccination data $q_{t}$ and norm $p_{t}$ according to (3).

\subsection{Political stability and dual channel of the Politico stimulation}

As can be seen from Fig. 3b), there were periods of time (in late January-early February, as well as in the second half of March) when the daily ratings were equal to 1 . In fact, these were the times when the Citizen was satisfied with the pace of vaccination. Regular citizen approval of
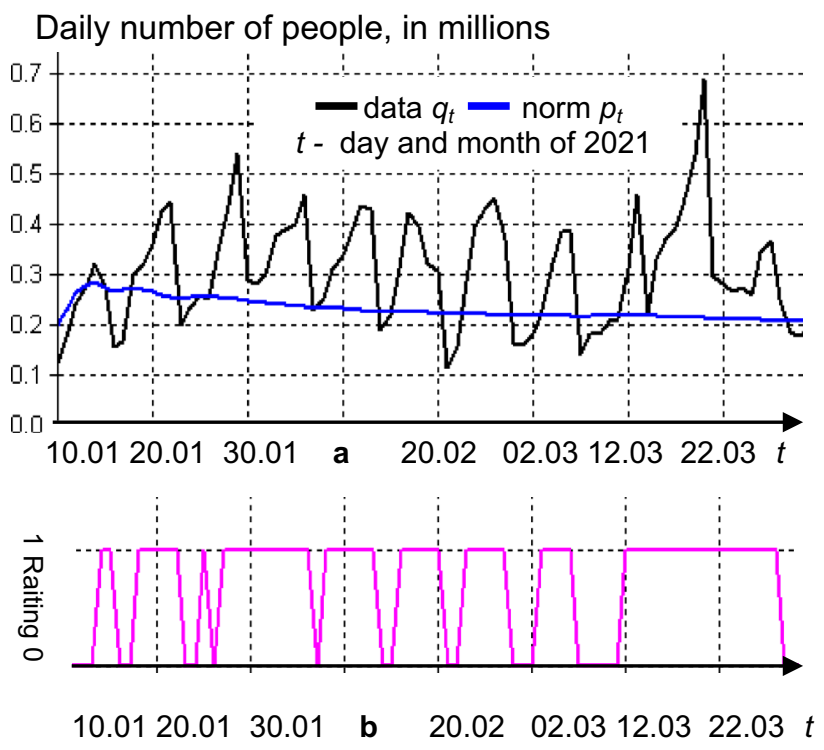

Fig. 3 a Daily number of people vaccinated $q_{t}$ (black line) and daily supply norm $p_{t}$ (blue line), in millions of people; $\mathbf{b}$ daily rating of the Politico $r_{t}$ (pink line) government action to provide life-saving vaccine fosters political stability.

Let us see how the mechanism $P=(Z, R)$ encourages the Politico to increase the pace of vaccination. First, if $q_{t} \geq p_{t}$, then $r_{t}=1$, and the Politico obtains rating 1 . Otherwise, the Politico obtains zero rating $\left(s_{t}=0\right)$. Therefore, the Politico rating is growing with $q_{t}$.

Second, according to (2), the higher the number of people vaccinated $q_{t}$, the lower norm $p_{\tau}$ for the future period $\tau, \tau=\overline{t+1, t+T}$. However, according to (3), this norm $p_{\tau}$ plays the role of the threshold value of number $q_{\tau}$ of people vaccinated in future period $\tau$ at which the Politico will receives rating 1 in this period $\tau, \tau=\overline{t+1, t+T}$. Consequently, it becomes easier for the Politico to get rating 1 in period $\tau$ even with a lower value of the random maximum supply $Q_{\tau}, \tau=\overline{t+1, t+T}$.

In other words, when the Citizen uses an optimal control mechanism $P=(Z, R)$, it stimulates the Politico to accelerate vaccination through two channels - today and in the future. Firstly, with an increase of the number of vaccinated people $\left(q_{t}\right)$, the Politico receives the best rating today. Second, the thresholds for getting better ratings of the Politico in the future are lowered. This means two-channel stimulation of the Politico in supplying vaccination.

Thus, mechanism $P=(Z, R)$ that satisfies the Statement 2 encourages the Politico to increase the number of people vaccinated. Then, the Politico is interested in taking full advantage of all the opportunities of supply of vaccine. In fact, digital mechanism $P=(Z, R)$ forms the progressive consciousness of the Politico in the interests of citizens, and aims to reconcile the interests of the Politico and society. This example illustrates the simplicity and transparency of Politico's control mechanism $P=(Z, R)$.

\section{Discussion}

In fact, the above considered a complex social system and the behavior of its main elements: citizens and politicians in a situation of crisis and uncertainty. Such situation in the process of the Pandemic regarding the availability of the vaccine in England is specifically described. It is assumed that the failure of the authorities to provide this product to the population in conditions of uncertainty may lead to political instability. Therefore, an adequate application of AI (with digital learning and adaptation) can help to strengthen stability and reduce uncertainties in this process.

\subsection{The added value of model using Al}

Concerned citizens can access information on vaccine supplies, vaccination rates from the media and official websites, 
and calculate vaccination indicators on their own using simple school math.

However, to form the optimal ratings of politicians in the face of uncertainty, citizens need special knowledge. To set up the optimal norm for assessing the performance of the authorities, knowledge from the theory of digital (machine) learning is required. To substantiate the procedure for forming the rating of a politician using this assessment norm, knowledge from the theory of organizational control is required. The mathematical model described above saves time and money for citizens who are not familiar with these theories to acquire such special knowledge. This provides the added value of using this AI-driven approach.

In our case, the use of AI helps people evaluate politicians better, more accurately, faster and cheaper than with traditional heuristic methods of information processing. After all, simple school mathematics is no longer enough to regularly obtain optimal adaptive ratings in the face of uncertainty associated with both random external influences and the possible undesirable activity of politicians.

The developed mechanism can be used by any citizen for permanent evaluation of policy with digital self-learning. Using AI to generate regular assessments of politician will not lead to high costs for citizens if such a mechanism is implemented as an application on their smart phones (see below, Sect. 6.3).

\subsection{Perspective models of a digital society}

Above, the simplest hierarchical model of a digital society consisting of the Citizen and the Politico (authorities) subject to random external influences is considered. The internal weakness of such digital society is related to the fact that the Politico can pursue personal, not public targets. Such undesirable activity of Politico introduces additional uncertainty, and influences the assessment of the actions of Politico by Citizen.

This is the crux of the problem. It is expected that the introduction of digital decision-making models should reduce uncertainty (associated with both random external influences and unwanted Politico activity) and ensure an adequate assessment of Politico by citizens. Deploying a specific AI system with digital learning and adaptive scoring optimization can help citizens evaluate policymakers' procurement and distribution of the vaccine in the face of uncertainty. In crisis situations, the challenge of holding politicians accountable becomes a vital to achieving stability.

The model of digital society is based on digital models of social actors - citizens and politicians, considered as rational subjects. Such reduced models are based on the behavioral school of psychology, and are similar to the model of "Homo economicus"- a rational economic agent pursuing, first of all, its own interests. In such behavioral models, individual human behavior and the functioning of complex societies are based on the assumption that people respond to physiological (hormonal), psychological, material, and economic stimuli, especially those that seem to satisfy material needs (biological and economic). Based on these premises, behavioral models assume that the actions and behavior of people, in their various aspects, can be explained, first of all, on the basis of satisfying their specific needs. However, such models of socio-political systems are constantly criticized for not reflecting the "mechanism of real complex social constructions", which also include symbolic social goals (beliefs), social roles, ethical orientation, group cohesion, etc.

Unfortunately, however, today, there are no digital models of such a "mechanism of real complex social constructions". On the other hand, today society needs to address uncertainty in the context of the Pandemic.

Therefore, when constructing the above-mentioned mathematical model, we had to abstract from real complex social constructions for a while. Based on the assumption of the rationality of social actors in a digital political context, this model was used to describe the characteristics and behavior of its main elements: citizens and politicians.

Deploying AI based on learning, adaptation, and optimization models can help reduce uncertainty (and, among other things, eliminate unwanted policy actions). This will allow the development of more effective digital control of public policy using digital adaptive and machine learning. Under certain conditions (Statement 2), the optimal mechanism can be developed in which a politician (official) fully uses its capabilities in the interests of the citizens.

Of course, this is a simplified mathematical model of the functioning of a digital society and the interaction between its parts (citizens, politicians, and officials). There, the politician is interested in increasing his digital rating from the public, which undermines his propensity to pursue purely personal goals. This means initiating action to increase the supply of vaccine. Thus, a certain digital balance is established between the main actors, improving political stability.

Basically, it boils down to having citizens monitor people being vaccinated on a daily basis. Through this, citizens set a specific norm by which a politician is judged: if vaccination falls below this norm, the rating is lowered, and vice versa. This, in turn, encourages politician to purchase enough vaccines to reduce the customizable norm at which it is priced. In general terms, it is a model based on meaningful interaction between citizens, politicians, officials, which guarantees "reward" to all parties for achieving balance. At this stage, such models can be accepted into circulation due to the inability today digitally simulate the aforementioned "mechanism of real complex social constructions."

On the other hand, traditionally, citizens, politicians, and officials play certain social roles, and not only pursue their own interests. Their digital counterparts are "complex social 
elements" that must, to one degree or another, correspond to the traditional standards of behavior of citizens, politicians and officials. Therefore, for a deeper study of decision-making and voting processes in a digital society, it is necessary to build and explore digital models of such "complex social elements".

Another possible direction for future research in digital society is associated with the formation of the above "real complex social constructions"-complex self-organizing systems based on the achievement of abstract symbolic social goals, such as beliefs, ethics, group cohesion, etc. After all, voting and evaluating politicians are, first of all, a social act, and not a mental or economic activity. With this in mind, the adequacy of advanced digital models of real complex socio-political systems should be assessed.

\subsection{Deployment of Al in social systems}

The salient concerns about the current shortcomings and fundamental flaws concerning deployment of AI in the public domain were considered in Sect. 1.3. Let us consider how these concerns are eliminated in relation to the above model, using examples (2), (3), and (7) of Spiegelhalter tests (see Sect. 1.3).

Test (2) Would something simpler, and more transparent and robust, be just as good?

In the model described above, the simplest and most transparent form of classifying the activities of a politician is used - the dichotomy, i.e., assigning the Politico one of two ratings - 0 or 1 . The reliability of this dichotomy is confirmed by the practice of democratic elections in which citizens vote for a politician (with rating 1) or against him (rating 0).

For simplicity, we also use linear loss functions, which are the most transparent and reliable. As a result, a citizen can calculate the norm according to formula (2) and the rating of the Politico according to formula (3), using school arithmetic.

Test (3) Could I explain how it works (in general) to anyone who is interested?

In general, model works very simple. With the help of an optimal self-learning algorithm, the current norm of evaluation of the Politico is adjusted, depending on the volume of vaccine supply. If the actual deliveries exceed this norm, then the Politico receives a higher current rating. Simultaneously, future norms for evaluating the Politico are decreasing. This makes it easier for a forward-thinking Politico to obtain higher ratings in the future, even under adverse accidental external influences. All this motivates the Politico in increasing the supply of vaccine.

Test (7) Does it actually help in practice?

As the introduction of AI, digital learning and ML systems has shown in the process of implementing the concept of Industry 4.0 in hierarchical industrial systems, this approach helps in practice, since it stimulates far-sighted leaders to increase their efficiency, for example, the volume of products (Tsyganov 2019). Developed optimal control mechanism $P=(Z, R)$ is very simple, and can be used by any citizen for permanent evaluation of a politician using digital self-learning. It is a simplest hybrid control mechanism based on statistics and correlation, as well as mathematical modeling and causation.

Concerns when using this mechanism in practice are mainly related to the reluctance or even inability to both learn how to use it and apply it constantly (for example, due to the lack of time for many citizens). In particular, concerns are associated with the definition by citizens of the parameters of the rationing procedure (2).

First, even with the simplest linear loss functions, citizen must set the coefficients $f$ and $m$, characterizing the ratio of losses of the first and second kind, respectively. To do this, citizen need to determine what is more important-to mistakenly support a careless politician or mistakenly not to support a conscientious politician.

Second, citizens must accept adaptation coefficients $\gamma_{t}, t=1,2, \ldots$. There is a solution that is acceptable to everyone: if citizens want to determine the norm of assessment of a politician as quickly as possible, then they must choose $\gamma_{t}=1 / t, \quad t=1,2, \ldots$ Then, according to Statement 1 , the rate of convergence of estimates (2) is maximum.

The possible way of deployment of optimal control mechanism $P=(Z, R)$ lies in the development of an application on a smart phone. With its help, it would be convenient for the citizen to set coefficients $f$ and $m$. After this, the current norms and ratings of a politician would be automatically calculated in real time.

With the mass installation of such an application on smart phones of citizens, politicians (authorities, officials) will have to constantly monitor these ratings and strive to increase them. And since in optimal control mechanism $P=(Z, R)$, the ratings increases with an increase in supply, politicians (authorities, officials) will strive to increase this supply, making the most of all emerging opportunities (including random ones).

In any case, when deploying AI, it is necessary to take into account warnings about too hasty use of AI, coming from both scientists and international organizations (see Sect. 1.3). Examples are the World Economic Forum White Paper (2019) on AI governance and EU Homepage (2020) on digital societies. AI deployment should address not only ethical constraints and adaptations, but also capacity and regulatory frameworks. In particular, the above White Paper on AI governance recommends careful consideration of ethical issues in attempts to deploy AI in social and political systems. They are of utmost importance-before embarking on a specific AI deployment. In this regard, in the future, a 
broader approach should be considered both to the specific problem under consideration and to the subject of AI deployment in general.

\section{Conclusions}

Political stability is determined by the loyalty of citizens. Therefore, with a vital commodity shortage, need to meet their needs quickly. For example, the Pandemic requires a vaccine supply system, structure, and mechanisms. To process big data, it is advisable to use such elements of $\mathrm{AI}$ as digital machine learning. However, this requires transparency and accountability of the use of AI in decision-making. To avoid abuse, modern control methods should be used in the event of conflicts of interest.

The paper illustrates a simple and transparent AIdriven approach to ensuring political stability when a vital commodity (such as the vaccine) is in short supply. This approach is based both on layered AI and the theory of organizational control. It is a hybrid approach based on statistics and correlation, as well as mathematical modeling and causation.

Further research in this direction can be associated with the system engineering of advanced AI-driven models of complex socio-political systems.

\section{References}

Abdulkareem M, Petersen S (2021) The promise of AI in detection, diagnosis and epidemiology for combating COVID-19: beyond the hype. Front Artif Intell 4:652669

Ahmed I, Ahmad M, Rodrigues J et al (2021) A deep learning-based social distance monitoring framework for COVID-19. Sustain Cities Soc 65:102571

Ahuja S, Reddy V, Marques O (2020) Artificial intelligence and COVID-19: a multidisciplinary approach. Integr Med Res 9:100434

Alazab M et al (2020) COVID-1019 prediction and detection using deep learning. Int J Comput Inf Syst Ind Manag Appl 12:168-181

Arifovic J, Ledyard J (2011) A behavioral model for mechanism design: individual evolutionary learning. J Econ Behav Organ 78:375-395

Auster S (2013) Asymmetric awareness and moral hazard. Games Econom Behav 82:503-521

Bagamaev R, Gurlev I, Klukvin S et al (2005) Adaptive mechanism for mastering capital and improving international stability. IFACPapersOnLine 16:42-45

Boddy C, Ladyshewsky R, Galvin P (2010) Leaders without ethics in global business: corporate psychopaths. $\mathrm{J}$ of Public Affairs 10:121-138

Burkov V, Gubko M, Korgin N, Novikov D et al (2013) Mechanism synthesis and management. NOVA Publishers, New York

De Laat P (2018) Algorithmic decision-making based on machine learning from big data: can transparency restore accountability? Philos Technol Spring 31:525-541

De Fine LK, de Fine LJ (2020) Artificial intelligence, transparency, and public decision-making. AI Soc 35:917-926

Diallo S, Shults F, Wildman W (2021) Minding morality: ethical artificial societies for public policy modeling. AI Soc 36:49-57
Fehr E, Rangel A (2011) Neuroeconomic foundations of economic choice - recent advances. J Econ Perspect 25:3-30

Gill KS (2020a) Prediction paradigm: the human price for instrumentalism. AI Soc 35:3

Gill KS (2020b) The trappings of AI agency. AI Soc 35:289-296

GOV.UK (2021) Coronavirus (COVID-19) in the UK. https://coron avirus.data.gov.uk/details/vaccinations, last accessed 2021/04/12

Groumpos PP (2021) Modelling COVID-19 using Fuzzy Cognitive Maps(FCM). EAI Endorsed Trans Bioeng Bioinform 1(2):e3, 1-13. https://doi.org/10.4108/eai.24-2-2021.168728.

EU Homepage (2020) Creating a digital society. https://ec.europa.eu/ digital-single-market/en/creating-digital-society, last accessed 2020/10/19

Islam M, Rahaman A, Islam M (2020) Development of smart healthcare monitoring system in IoT environment. SN Comput Sci 1:185

Jamshidi M, Lalbakhsh A, Talla J et al (2020) Artificial intelligence and COVID-19: deep learning approaches for diagnosis and treatment. IEEE Access 8:109581-109595

Kossiakoff A, Sweet W, Seymour S et al (2011) Systems engineering. Principles and practice. Wiley, New York

Lipman-Blumen J (2004) The allure of toxic leaders. Oxford University Press, Oxford

Mohammad-Rahimi N, Ghalyanchi-Langeroudi T, Ghafouri F (2021) Application of machine learning in diagnosis of COVID-19 through X-ray and CT images: a scoping review. Front Cardiovasc Med 8:638011

Naudé W (2020) Artificial intelligence vs COVID-19: limitations, constraints and pitfalls. AI Soc 35:1-5

Nguyen T (2020) Artificial intelligence in the battle against coronavirus (COVID-19): a survey and future research directions. ArXiv: 200807343v1 [csCY]

Recht B (2020) Reflections on the learning-to-control renaissance. In: Proc. of the 21st IFAC World Congress. Elsevier, Berlin, p 4707

Schultz V, Tsyganov V (2009) Oligarchy, ontology, cycles, and change in a globalizing world. Sotsiologicheskie Issledovaniya 2:3-15

Schultz V, Tsyganov V (2012) Humanitarian high technologies in political system of society. Sotsiologicheskie Issledovaniya 8:85-93

Shinde G, Kalamkar A, Mahalle P et al (2020) Forecasting models for coronavirus disease (COVID-19): a survey of the state-of-the-art. SN Comput Sci 1:197

Simon H (1966) Models of man-social and rational. Wiley, New York

Soros G (1998) The crisis of global capitalism. Little, Brown \& Company, Boston

Spiegelhalter D (2020) Should we trust algorithms? Harvard Data Sci Rev 2:1

Tayarani N (2021) Applications of artificial intelligence in battling against COVID-19: a literature review. Chaos Solitons Fractals $142: 110338$

Tsyganov V (2014) Limits of global growth, stagnation, creativity, and international stability. AI Soc 29:259-266

Tsyganov V (2019) Designing adaptive information models for production management. Procedia CIRP 84:1088-1093

Tsyganov V (2020) Socio-political stability, voter's emotional expectations, and information management. AI Soc. https://doi.org/10. 1007/s00146-020-01017-8

Tversky A, Kahneman D (1992) Advances in prospect theory: cumulative representation of uncertainty. J Risk Uncertain 5:297-323

White Paper of World Economic Forum (2019) AI governance: a holistic approach to implement ethics into AI. World Economic Forum Homepage, https://www.weforum.org, last accessed 2021/04/12

Publisher's Note Springer Nature remains neutral with regard to jurisdictional claims in published maps and institutional affiliations. 